\title{
Do Urine Cultures for Urinary Tract Infections Decrease Follow-up Visits?
}

\author{
Jeremy Daniel Johnson, MD, MPH, Heather M. O'Mara, DO, Hyrum F. Durtschi, DO, \\ and Branko Kopjar, MD PhD, MS
}

Background: No major clinical practice guideline recommends ordering a urine culture in the management of uncomplicated urinary tract infections (UTIs). In this era of increasing antibiotic resistance, our objective was to determine if ordering urine cultures for adult women with uncomplicated UTIs provides results that lead to a decrease in follow-up visits for continued UTI symptoms.

Methods: This was a retrospective cohort study from the outpatient family medicine clinic, Madigan Army Medical Center, Tacoma, WA. Seven hundred seventy-nine nondiabetic, nonpregnant, adult (aged 18-65 years) female patients (mean age \pm SD, 32.9 \pm 12.1 years) diagnosed with a UTI from November 2006 through March 2008. The main outcome measure was follow-up outpatient visits within 2 weeks for recurrent UTI symptoms.

Results: Of the 779 patients studied, $332(43 \%)$ had no urine culture ordered as part of their management and $447(57 \%)$ had a urine culture ordered. There was no significant difference in the rate of follow-up within 2 weeks for continued UTI symptoms between the cohort without urine culture (28 of $332 ; 8.4 \%)$ and the cohort with urine culture $(39$ of $447 ; 8.7 \%)\left(\chi^{2}=0.021 ; P=.89\right)$. In multivariate logistic regression, ordering a urine culture was not associated with a decreased rate of follow-up visits (adjusted odds ratio, 1.11 ; 95\% CI, 0.65-1.90).

Conclusions: In adult women with uncomplicated UTI, ordering a urine culture was not found to be associated with a decrease in follow-up clinic visits. This finding is consistent with current guidelines that recommend against ordering a urine culture for uncomplicated UTIs. (J Am Board Fam Med 2011; 24:647-655.)

Keywords: Follow-up, Urine Cultures, Urinary Tract Infections

Uncomplicated urinary tract infections (UTIs), which include acute cystitis, are one of the most common presenting complaints in primary care. In 1997, UTIs accounted for seven million office visits and one million emergency department visits in the United States. ${ }^{1}$ Numerous worldwide publications and practice guidelines have recommended against

This article was externally peer reviewed.

Submitted 18 December 2010; revised 17 April 2011; accepted 20 April 2011.

From the Department of Family Medicine, Tripler Army Medical Center, Honolulu, HI (JDJ); the Second Brigade Combat Team, 82nd Airborne Division, Ft. Bragg, NC (HMO); the Grafenwoehr Army Health Clinic, Grafenwoehr, Germany (HFD); and the Department of Health Services, University of Washington, Seattle, WA (BK).

Funding: Financial and material support provided by the United States Army.

Conflict of interest: none declared.

Disclaimer: The US Army had no role in determining study design, data collection, analysis, or interpretation of data. The US Army had no role in writing or submitting the manuscript. the use of routine urine cultures in uncomplicated UTIs for cost-effectiveness reasons. ${ }^{2-12}$ Despite these recommendations, one study found that primary care physicians (family medicine, general internal medicine, obstetrics/gynecology, and emergency medicine) ordered urine cultures $30 \%$ of the time in the management of uncomplicated UTIs, and another study found that they ordered urine cultures $32 \%$ to $57 \%$ of the time depending on location and patient's insurance type. ${ }^{13,14}$

Corresponding author: Jeremy D. Johnson, MD, MPH, Tripler Army Medical Center, Attn: MCHK-FMR, 1 Jarrett White Road, Honolulu, HI 96734. (E-mail: jeremy. daniel.johnson@us.army.mil).

See Related Article on Page 628. 
In 1984 Dr. Henry Schultz and his colleagues ${ }^{15}$ published a prospective study of UTIs in a primary care acute illness service. They argued that, for conventionally managed UTIs, because medications accounted for only $10 \%$ to $15 \%$ of the cost and physician fees only $22 \%$, any cost savings would have to come from a reduction in the number of laboratory tests. Although this conclusion may have been valid at the time of the study, its validity today may be mitigated by rising antibiotic resistance, changing prescribing patterns, cost of antibiotics, and other factors. Schultz et $\mathrm{al}^{15}$ found that clinical symptoms of frequency, urgency, and dysuria seemed to be better guides to management than urine cultures, and urine culture susceptibility testing did not contribute to the management of UTIs. Significant limitations to the study were that all patients received a urine culture and no urine cultures revealed bacteria resistant to the antibiotic used for treatment, which is far from the current situation of drug-resistant bacteria. Since the 1984 Schultz et $\mathrm{al}^{15}$ study, no major clinical practice guideline, cost-effectiveness analysis, or medical society has recommended a urine culture as part of the management of an uncomplicated UTI. ${ }^{5,16-21}$ No study in the published literature has yet looked at whether ordering a urine culture at the time of diagnosis and treatment of UTI decreases follow-up visits.

Our study evaluated the current practice at three family medicine clinics at Madigan Army Medical Center in Tacoma, Washington, through empiric study of 779 patients with presumed uncomplicated UTIs. This retrospective cohort study looked at the percentage of instances in which providers ordered urine cultures for patients with uncomplicated UTIs, and it evaluated the patients' management to see if obtaining a urine culture decreased follow-up visits for continued UTI symptoms. Secondary outcomes were based on the questions, (1) Does ordering a urine culture increase the percent of patients who have a second antibiotic prescribed within 2 weeks? and (2) Does ordering a urine culture decrease the percent of patients who call their provider with a complaint of continued UTI symptoms?

\section{Methods}

\section{Study Sample}

After Madigan Army Medical Center Institutional Review Board and University of Washington $\mathrm{Hu}-$ man Subjects Review Committee approval, study participants were obtained through an electronic search of the hospital's electronic medical record (EMR). Inclusion criteria for the study were (1) female sex; (2) age 18 to 65 years; (3) assigned to family medicine at Madigan Army Medical Center; (4) seen in the family medicine clinic from November 1, 2006, to March 25, 2008; and 5) diagnosed with a UTI or acute cystitis.

The exclusion criteria were (1) being pregnant; (2) being diabetic; (3) being diagnosed with a UTI or cystitis during the preceding 6 weeks; (4) being treated with an antibiotic during the preceding 6 weeks; (5) the inability to access medical records to confirm inclusion and exclusion criteria; and (6) having any mention by the medical provider seeing the patient that the patient has a medical condition that qualifies the patient as having a complicated UTI (eg, severe kidney disease).

Because pregnant and diabetic patients are at higher risk for complications from UTI, and because the literature has traditionally excluded one or both of them from studies of uncomplicated UTIs, we also excluded them..$^{2,15,21-23} \mathrm{We}$ decided to exclude those patients diagnosed with a UTI during the preceding 6 weeks and those treated with an antibiotic during the preceding 6 weeks to avoid patients who may have been incompletely treated or who may have a higher chance of resistant organisms involved with the current infection.

\section{Study Procedures}

All patient visits from Madigan's three family medicine clinics were searched electronically to find an International Classification of Diseases, 9th Revision (ICD-9) code match for UTI (ICD-9 code 599.0) and acute cystitis (ICD-9 code 595.0). Patients with either of these two diagnoses who met inclusion criteria and were not excluded based on exclusion criteria were considered to have an uncomplicated UTI for the purposes of the study. The search also was limited to female patients in the study's age range of 18 to 65 years. Patients who had an ICD-9 code diagnosis of UTI or acute cystitis during the previous 42 days (6 weeks) were eliminated according to the third exclusion criteria mentioned above. Patients meeting inclusion criteria were evaluated by one of three researchers using the hospital's EMR.

Every patient had her entire medical visit note from her UTI visit, the EMR list of all diagnoses 
ever given the patient, the EMR list of all medications ever prescribed to the patient and whether they were dispensed, and her EMR demographic information reviewed for exclusion criteria and all data elements required by the study.

All follow-up visits or phone calls in the 2 weeks after the initial UTI visit, which were recorded electronically, were reviewed. Only those follow-up visits or patient-initiated phone calls in which the subject specifically complained about continued UTI symptoms (dysuria, frequency, urgency, or, rarely, suprapubic pain) were counted toward the study outcomes.

Participants' or their spouses' listed military rank was used as a marker for socioeconomic status. Rank was dichotomized as lower and higher socioeconomic status. To be consistent with previous studies, lower socioeconomic status included the enlisted ranks of enlisted 1 through 4 , and all other ranks were considered higher socioeconomic status. $^{24,25} \mathrm{We}$ used the four racial categories that are found in the EMR demographics section: white, African American, Asian/Pacific Islander, and other race.

\section{Statistical Analysis}

Data were collected using SPSS statistical software version 14.0 (SPSS, Inc., Chicago, IL). All patients with a diagnosis of UTI or acute cystitis during the approved study period were reviewed in alphabetical order (to exclude duplicates). We reviewed 1199 records before stopping per institutional review board requirements because we had enough patients to meet $80 \%$ power, which left 241 patients unevaluated. Data quality was assured through several audits. We had greater than $98.5 \%$ accuracy on all audits. All errors discovered in the audits were corrected before data analysis.

The design of this study was a retrospective cohort study. Power analysis was done at the beginning of the study. We wanted to see if ordering a urine culture would decrease follow-up visits from $8.7 \%$ to $3.7 \%$ (a $5 \%$ drop), which we thought would be clinically meaningful. Our sample size of 779 patients provided a power of $81 \%$ at an $\alpha$ error level of $5 \% .^{26,27}$

The statistical method used to evaluate the main outcome measure (Is ordering a urine culture associated with a decrease in follow-up visits?) was the $\chi^{2}$ test with a two-tailed $P=.05$. Multivariate logistic regression was also used with covariates of age (continuous scale); provider status (allopathic/ osteopathic physicians [doctor of medicine/doctor of osteopathic medicine] vs midlevel provider); clinic; socioeconomic status; whether medication prescription was filled at the pharmacy; initial antibiotic used to treat the UTI; urine culture ordered; participant status as active duty soldier or civilian family member; and trimethoprim/sulfamethoxazole (TMP-SMX) allergy. Because race data were available in only $65 \%$ (512 of 779 ) of the patients, we did not use race in the multivariate analysis; it would have excluded $35 \%$ of the patients from the analysis. When race was added to the multivariate analysis, the results were unchanged overall. When fever, costovertebral angle tenderness, and nausea were included in the multivariate analysis, 331 cases had to be excluded because of missing data; thus, we did not include them in the final multivariate analysis. Of note, however, when these three characteristics were added, the results were unchanged overall.

\section{Results}

We reviewed the records of 1199 patients. We excluded 420 and were left with 779 patients (Figure 1). Of the 779 patients studied, 332

Figure 1. Patient exclusions. *Improperly coded diagnoses included asymptomatic bacteruria, history of urinary tract infection (UTI), abdominal pain, vaginal complaint, pyelonephritis, and upper respiratory infection. **Complicated U'TIs included patients with Wilms tumor, hydronephrosis and chronic renal failure, solitary kidney, lymphoma, renal stent, small kidney, and unspecified genetic disorder causing UTIs.

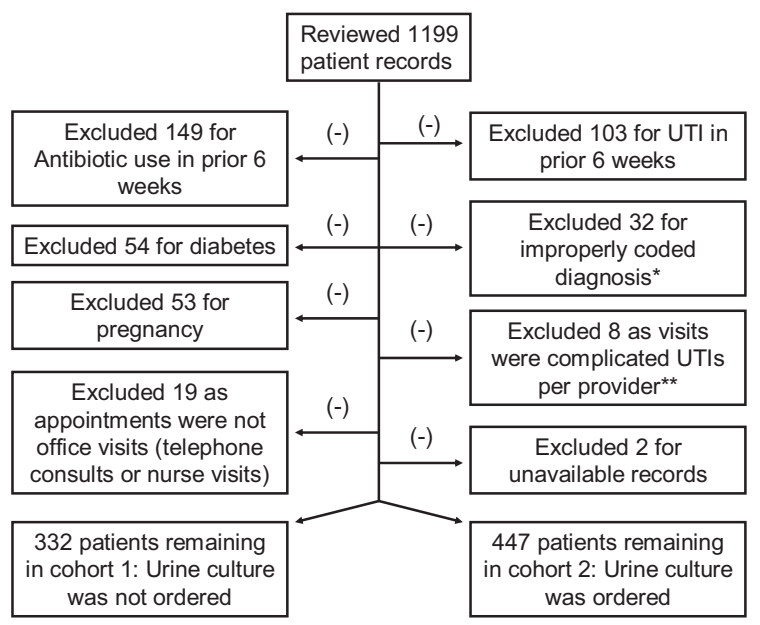


Table 1. Demographic Characteristics of Study Cohorts

\begin{tabular}{|c|c|c|c|c|}
\hline Characteristic & $\begin{array}{l}\text { Total Population } \\
(\mathrm{N}=783)\end{array}$ & $\begin{array}{l}\text { Cohort } 1^{*} \\
(\mathrm{n}=332)\end{array}$ & $\begin{array}{l}\text { Cohort } 2^{+} \\
(\mathrm{n}=447)\end{array}$ & $P$ \\
\hline Average age (mean years \pm SD) & $32.9 \pm 120.1$ & $32.4 \pm 11.8$ & $33.2 \pm 12.3$ & .38 \\
\hline Race $^{\ddagger}$ & & & & .53 \\
\hline White & 325 & $67 \%$ & $61 \%$ & \\
\hline African American & 67 & $13 \%$ & $13 \%$ & \\
\hline Asian/Pacific Islander & 42 & $7 \%$ & $9 \%$ & \\
\hline Other & 78 & $14 \%$ & $16 \%$ & \\
\hline Socioeconomic status ${ }^{\ddagger}$ & & & & .26 \\
\hline Lower & 243 & $33 \%$ & $30 \%$ & \\
\hline Higher & 534 & $67 \%$ & $70 \%$ & \\
\hline Active duty soldier & 193 & $29 \%$ & $22 \%$ & .02 \\
\hline
\end{tabular}

${ }^{*}$ No urine culture ordered.

${ }^{\dagger}$ Urine culture ordered.

${ }^{\ddagger}$ Some patients were missing data from these variables: race, $\mathrm{n}=267$; socioeconomic status, $\mathrm{n}=2$. Unpaired $t$ test was used to compare means, $\chi^{2}$ test was used to compare proportions (missing data excluded).

(43\%) had no urine culture ordered during the management of their UTI (cohort 1), and 447 (57\%) did have a urine culture ordered during the management of their UTI (cohort 2). Table 1 lists demographic characteristics for cohorts 1 and 2. Table 2 lists clinical characteristics for cohorts 1 and 2 .

\section{Primary and Secondary Outcome Measures}

When evaluating our primary hypothesis, we found the follow-up rate for cohort 1 (no urine culture) was 28 of 332 patients $(8.4 \%)$, whereas the follow-up rate for cohort 2 (urine culture obtained) was 39 of 447 patients $(8.7 \%)$. The $\chi^{2}$ test showed no statistically significant difference between the

Table 2. Clinical Characteristics of Study Cohorts

\begin{tabular}{|c|c|c|c|c|}
\hline Characteristic & $\begin{array}{l}\text { Total Population } \\
\quad(\mathrm{N}=783)\end{array}$ & $\begin{array}{l}\text { Cohort } 1^{*} \\
(\mathrm{n}=332)\end{array}$ & $\begin{array}{l}{\text { Cohort } 2^{+}}_{(\mathrm{n}=447)}\end{array}$ & $P$ \\
\hline Clinic seeing patient & & & & $<.001$ \\
\hline Hospital clinic & 554 & $66 \%$ & $75 \%$ & \\
\hline Community clinic \#1 & 126 & $15 \%$ & $17 \%$ & \\
\hline Community clinic \#2 & 99 & $20 \%$ & $7 \%$ & \\
\hline Seen by midlevel provider (PA/NP), not physician & 542 & $74 \%$ & $66 \%$ & .02 \\
\hline Initial antibiotic used & & & & .01 \\
\hline TMP-SMX & 411 & $58 \%$ & $49 \%$ & \\
\hline Fluoroquinolone & 149 & $20 \%$ & $19 \%$ & \\
\hline Nitrofurantoin & 187 & $19 \%$ & $28 \%$ & \\
\hline Other medicine & 24 & $2 \%$ & $4 \%$ & \\
\hline No medication prescribed & 8 & $0.3 \%$ & $1.6 \%$ & \\
\hline Allergic to TMP-SMX & 91 & $12 \%$ & $11 \%$ & .78 \\
\hline Fever at initial visit ${ }^{\ddagger}$ & 1 & $0 \%$ & $0.2 \%$ & 1.0 \\
\hline Nausea or vomiting ${ }^{\ddagger}$ & 62 & $6 \%$ & $9 \%$ & .27 \\
\hline Flank pain or CVA tenderness ${ }^{\ddagger}$ & 115 & $13 \%$ & $16 \%$ & .61 \\
\hline
\end{tabular}

*No urine culture ordered.

${ }^{\dagger}$ Urine culture ordered.

${ }^{\ddagger}$ Some patients ( $\mathrm{n}$ ) were missing data from these variables: fever, $\mathrm{n}=32$; nausea/vomiting, $\mathrm{n}=221$; flank pain/CVA tenderness, $\mathrm{n}=$ 172. Unpaired $t$ test was used to compare means. $\chi^{2}$ test was used to compare proportions (missing data excluded), and Fisher's exact test was used for fever.

PA, physician's assistant; NP, nurse practitioner; TMP-SMX, trimethoprim/sulfamethoxazole; CVA, costovertebral angle. 
Table 3. Did Ordering a Urine Culture Change Outcomes?

\begin{tabular}{lccc}
\hline Outcome & Cohort 1* $(\%)(\mathrm{n}=332)$ & Cohort 2 $(\%)(\mathrm{n}=447)$ & Pearson $\chi^{2}$ result \\
\hline Follow-up visit within 2 weeks & 8.4 & 8.7 & 0.02 \\
New antibiotic ordered within 2 weeks & 6.6 & 8.9 & .89 \\
Patient-initiated telephone consult for continued & 1.8 & 3.6 & .24 \\
$\quad$ symptoms within 2 weeks & & .14 \\
\hline
\end{tabular}

*No urine culture ordered.

${ }^{\dagger}$ Urine culture ordered.

groups $\left(\chi^{2}=0.02 ; P=.89\right.$; Table 3$)$. Similarly, there was no statistically significant difference between the cohorts when looking at either a second antibiotic being prescribed within 2 weeks of the initial visit or a telephone consult for continued UTI symptoms within 2 weeks of the initial visit, though the study was not powered for these outcomes (Table 3).

\section{Urine Culture Results}

Of the 447 urine cultures ordered, 243 (54\%) grew $\geq 1,000$ colony-forming units per milliliter of a specific bacteria, whereas 101 (23\%) had no growth and 94 (21\%) grew mixed contaminants only (see Appendix 1 for isolates and resistance rates). The Escherichia coli resistance to TMP-SMX in our sample was $13 \%$ (similar to $15 \%$ for our hospital).

Of all 447 urine cultures ordered, only 21 (5\%) grew bacteria that were resistant to the antibiotic prescribed (17 TMP-SMX, one fluoroquinolone, two amoxicillin, one nitrofurantoin), and one culture $(0.2 \%)$ showed intermediate resistance to the medication prescribed (nitrofurantoin). Nine of these 21 patients did not follow-up for continued symptoms because they were either contacted by their provider to change their medication $(n=6)$ or they presumably self resolved $(\mathrm{n}=3)$. The other patients $(n=12)$ followed up for continued UTI symptoms and had their antibiotic changed, but most $(n=7)$ did not have urine culture results by the time they followed up. One patient in the urine culture group was initially sent home after her outpatient visit, but returned to the emergency department later the same day with inability to tolerate liquids. She was then admitted to the hospital with a diagnosis of pyelonephritis.

\section{Bivariate and Multivariate Testing}

Because ordering a urine culture did not seem to have any significant relationship with obtaining a follow-up visit (see Table 3), we tested all variables in our model with bivariate methods to determine if any had a significant relationship to a patient obtaining a follow-up visit for continued UTI symptoms. Of all variables tested, only having a urine culture that grew an organism that was resistant to the antibiotic prescribed showed a significant relationship to obtaining a follow-up visit for continued UTI symptoms (Table 4). On multivariate analysis using the enter method, ordering a urine culture did not decrease the odds of a follow-up visit (adjusted odds ratio, 1.11; 95\% CI, $0.65-1.90)$. The variable for urine culture growing a resistant organism was not added to the multivariate logistic regression because it would have excluded patients who had no urine culture performed (see Table 5).

\section{Discussion}

In our study sample of 779 patients, the patients who had a urine culture ordered as part of their management did not show any significant decrease in follow-up visits compared with those who did not have a urine culture ordered. Similarly, there was no statistically significant difference between the cohorts when looking at either a second antibiotic being prescribed within 2 weeks of the initial visit or a telephone consult for continued UTI symptoms within 2 weeks of the initial visit, though the study was not powered for these outcomes. Multivariate analysis showed that ordering a urine culture was not associated with a significant change in follow-up rates.

This study had several strengths. To gather data, we used electronic patient records, which allowed us to track all outpatient visits, emergency department visits, inpatient visits, pharmacy prescriptions, ancillary tests, and telephone consults. We studied a facility with a very large patient population of 60,000 enrollees, which provided an ade- 
Table 4. Bivariate Testing: Patients Who Followed Up Within 2 Weeks for Continued Urinary Tract Infection Symptoms $(\mathbf{n}=779)$

\begin{tabular}{|c|c|c|c|}
\hline Characteristic & Total Patients (n [\%]) & $\begin{array}{c}\text { Patients Who Followed Up Within } 2 \\
\text { Weeks for UTI Symptoms (\%) }\end{array}$ & $P$ \\
\hline Average age (mean years \pm SD) & $32.9 \pm 12.1$ & $31.3 \pm 11.8$ & .28 \\
\hline Race $^{*}$ & & & .30 \\
\hline White & $325(64)$ & 10 & \\
\hline African American & $67(13)$ & 4 & \\
\hline Asian/Pacific Islander & $42(8)$ & 5 & \\
\hline Other & $78(15)$ & 11 & \\
\hline Socioeconomic status & & & .16 \\
\hline Lower & $243(31)$ & 11 & \\
\hline Higher & $534(69)$ & 8 & \\
\hline Clinic seeing patient & & & .62 \\
\hline Hospital based clinic & $554(71)$ & 8 & \\
\hline Community based clinic \#1 & $126(16)$ & 9 & \\
\hline Community based clinic \#2 & $99(13)$ & 11 & \\
\hline Seen by midlevel provider (PA/NP) not physician & $542(70)$ & 9 & .91 \\
\hline Initial antibiotic used & & & .48 \\
\hline TMP-SMX & $411(53)$ & 9 & \\
\hline Fluoroquinolone & $149(19)$ & 6 & \\
\hline Nitrofurantoin & $187(24)$ & 11 & \\
\hline Other medicine & $24(3)$ & 13 & \\
\hline No medication prescribed & $8(1)$ & 0 & \\
\hline Active duty soldier & $193(25)$ & 11 & .11 \\
\hline Allergic to TMP-SMX & $91(12)$ & 10 & .64 \\
\hline Fever at initial visit & $1(0.1)$ & 0 & 1.00 \\
\hline Nausea or vomiting* & $62(11)$ & 15 & .08 \\
\hline Flank pain or CVA tenderness* & $115(19)$ & 13 & .14 \\
\hline Medication filled at pharmacy & $751(97)$ & 9 & .69 \\
\hline Urine culture obtained & $447(57)$ & 9 & .89 \\
\hline Urine culture not obtained & $332(43)$ & 8 & \\
\hline Urine culture results & & & .70 \\
\hline Urine culture grew specific organism & $243(54)$ & 9 & \\
\hline Urine culture grew mixed flora & $94(21)$ & 11 & \\
\hline Urine culture had no growth & $110(25)$ & 7 & \\
\hline Urine culture resistance to prescribed antibiotic & & & $<.001$ \\
\hline Organism resistant & $21(10)$ & 52 & \\
\hline Organism not resistant & $200(90)$ & 5 & \\
\hline
\end{tabular}

*Some patients were missing data from these variables: race, $\mathrm{n}=267$; socioeconomic status, $\mathrm{n}=2$; fever, $\mathrm{n}=32$; nausea/ vomiting, $\mathrm{n}=221$; flank pain/CVA tenderness, $\mathrm{n}=172$. Unpaired $t$ test was used to compare means, and $\chi^{2}$ test was used to compare proportions (missing data excluded).

UTI, urinary tract infection; PA, physician's assistant; NP, nurse practitioner; NS, Not significant; TMP-SMX, trimethoprim/ sulfamethoxazole; CVA, costovertebral angle.

quate sample size over a relatively short period of time. We studied a facility where all medical care is provided free to all patients, which helped ensure that patients would not avoid follow-up visits because of the lack of ability to pay. Finally, we used a medical system that electronically records when a patient calls for a follow-up visit even if no visit is available.
Study weaknesses include the fact that this study was not a randomized controlled trial, but rather a cohort trial, so causality cannot be determined. Another limitation is that Madigan Hospital has a $15 \%$ E. coli resistance to the antibiotic TMP-SMX. The E. coli resistance levels vary around the world, and thus the results of this study may not apply to other populations. This study was conducted in 
Table 5. Multivariate Logistic Regression: Which Factors Are Related to Following Up Within 2 Weeks for Continued Urinary Tract Infection Symptoms?

\begin{tabular}{lc}
\hline Characteristic & $\begin{array}{c}\text { Odds } \\
\text { ratio }(95 \% \mathrm{CI})\end{array}$ \\
\hline $\begin{array}{l}\text { Age (years) } \\
\text { Socioeconomic } \\
\text { status (senior rank) }\end{array}$ & $0.99(0.97-1.02)$ \\
Clinic seeing patient & $0.81(0.43-1.53)$ \\
$\begin{array}{l}\text { Initial antibiotic used } \\
\text { Civilian family member as } \\
\text { opposed to active duty } \\
\text { soldier }\end{array}$ & $\mathrm{NS}$ \\
$\begin{array}{l}\text { Seen by midlevel } \\
\text { provider (PA/NP) not } \\
\text { physician }\end{array}$ & $0.70(0.39-1.26)$ \\
$\begin{array}{l}\text { Allergic to TMP-SMX } \\
\text { Medication filled at } \\
\text { pharmacy }\end{array}$ & $1.05(0.59-1.85)$ \\
Urine culture ordered & $1.30(0.58-2.94)$ \\
\hline
\end{tabular}

PA, physician's assistant; NP, nurse practitioner; NS, not significant; TMP-SMX, trimethoprim/sulfamethoxazole.

three clinics in a military medical system, so results may not be generalizable. Race data were not always recorded in our EMR, yielding only $512(67 \%)$ patients with specified race. Because of this, we could not use race as a factor in our multivariate analysis, although race data were not significant in our bivariate analysis (sees Table 4 and 5). A final potential limitation is that we excluded patients that had UTIs in the prior 6 weeks. This may limit this study's applicability to patients with recurrent UTIs and very short asymptomatic intervals.

To our knowledge, this is the first published study looking specifically at whether ordering a urine culture at the time of diagnosis and treatment is associated with a decrease in follow-up rates for recurrent UTI symptoms. Several studies have recommended against ordering a urine culture based on assumptions of the effectiveness of urine cultures in decreasing symptom days or in looking at the number of resistant organisms that grew in cultures, but no other studies observed actual practice to determine the effectiveness of a urine culture in decreasing follow-up visits. ${ }^{2,15}$ Our study agrees with a recent UK prospective cohort study in which a urine culture was performed for all patients and found that trimethoprim resistance was only $13.9 \%$. This UK study found that 23 women required a urine culture to prevent one follow-up visit from resistance-based failure; thus, empiric treatment with no urine culture was recommended. ${ }^{10}$ Our study had a similar TMP-SMX resistance rate and a similar outcome; however, only one cohort in our study had a urine culture ordered. We had a control group and a test group, which allowed us to perform statistical tests to determine if a urine culture made a difference.

This study agrees with the current body of literature that recommends against ordering a urine culture in this population. In our study, ordering a urine culture was not associated with a change in outcomes for patients, as evidenced by follow-up visits, but it did result in increased laboratory costs of $\$ 5,546.75$, or $\$ 12.41$ per patient. This study shows a large variation in practice patterns by providers at a single hospital, and it reinforces the limited effectiveness of the urine culture as a means to identify resistant organisms and help prevent follow-up visits. It is our feeling that clinicians should follow current guidelines by avoiding routine urine cultures when treating uncomplicated UTIs, and instead order a urine culture only to confirm the diagnosis of UTI in doubtful cases before treatment or when initial UTI management fails. Policymakers should also consider these results when evaluating the cost effectiveness of laboratory policies of conducting urine cultures reflexively on patients with a positive urinalysis.

Although our study found no association between ordering a urine culture in the management of uncomplicated UTI and outcomes in terms of decreased follow-up visits and decreased patientinitiated phone calls for continued symptoms, a randomized controlled trial would be ideal. A randomized controlled trial may allow for a more comprehensive cost-benefit analysis. This study should be replicated in settings in which $E$. coli resistance to TMP-SMX is greater than $15 \%$ to see if the urine culture in those settings is also ineffective at preventing follow-up visits. Future studies should also be conducted in facilities where patient race is always recorded because this could possibly have an effect that we could not find because we had race data on only $67 \%$ of our participants.

The authors would like to thank the late Walter E. Stamm for the advice and guidance he provided to us during the creation and completion of this research study. 


\section{References}

1. Foxman B. Epidemiology of urinary tract infections: incidence, morbidity, and economic costs. Am J Med 2002;113(Suppl 1A):5S-13S.

2. Carlson KJ, Mulley AG. Management of acute dysuria. A decision-analysis model of alternative strategies. Ann Intern Med 1985;102(2):244-9.

3. Fenwick EA, Briggs AH, Hawke CI. Management of urinary tract infection in general practice: a cost-effectiveness analysis. Br J Gen Pract 2000; 50(457):635-9.

4. Hooton TM. Practice guidelines for urinary tract infection in the era of managed care. Int J Antimicrob Agents 1999;11(3-4):241-5; discussion 261-4.

5. Hooton TM. The current management strategies for community-acquired urinary tract infection. Infect Dis Clin North Am 2003;17(2):303-32.

6. Stamm WE, Hooton TM. Management of urinary tract infections in adults. N Engl J Med 1993; 329(18):1328-34.

7. Werman HA, Brown CG. Utility of urine cultures in the emergency department. Ann Emerg Med 1986; 15(3):302-7.

8. Colgan R, Hyner S, Chu S. Uncomplicated urinary tract infections in adults. In: Grabe $M$, Bishop MC, Bjerklund-Johansen TE, et al., eds. Guidelines on Urological Infections. Arnhem, The Netherlands: European Association of Urology; 2009:11-38.

9. Hummers-Pradier E, Ohse AM, Koch M, Heizmann WR, Kochen MM. Management of urinary tract infections in female general practice patients. Fam Pract 2005;22(1):71-7.

10. McNulty CA, Richards J, Livermore DM, et al. Clinical relevance of laboratory-reported antibiotic resistance in acute uncomplicated urinary tract infection in primary care. J Antimicrob Chemother 2006;58(5):1000-8.

11. van den Broek PJ, van Everdingen JJ, Dutch Institute for Quality Assurance. ["Urinary tract infections"revised CBO guideline.] Ned Tijdschr Geneeskd 1999; 143(49):2461-5.

12. McLaughlin SP, Carson CC. Urinary tract infections in women. Med Clin North Am 2004;88(2):417-29.

13. Wigton RS, Longenecker JC, Bryan TJ, Parenti C, Flach SD, Tape TG. Variation by specialty in the treatment of urinary tract infection in women. J Gen Intern Med 1999;14(8):491-4.
14. Carey TS, Weis K. Diagnostic testing and return visits for acute problems in prepaid, case-managed Medicaid plans compared with fee-for-service. Arch Int Med 1990;150(11):2369-72.

15. Schultz HJ, McCaffrey LA, Keys TF, Nobrega FT. Acute cystitis: a prospective study of laboratory tests and duration of therapy. Mayo Clin Proc 1984;59(6):391-7.

16. Czaja CA, Hooton TM. Update on acute uncomplicated urinary tract infection in women. Postgrad Med 2006;119(1):39-45.

17. Forland M. Urinary tract infection. How has its management changed? Postgrad Med 1993;93(5): 71-4, 77-8, 84-6.

18. Hooton TM, Stamm WE. Diagnosis and treatment of uncomplicated urinary tract infection. Infect Dis Clin North Am 1997;11(3):551-81.

19. Miller LG, Tang AW. Treatment of uncomplicated urinary tract infections in an era of increasing antimicrobial resistance. Mayo Clin Proc 2004;79(8): 1048-53; quiz 1053-4.

20. O'Connor PJ, Solberg LI, Christianson J, Amundson G, Mosser G. Mechanism of action and impact of a cystitis clinical practice guideline on outcomes and costs of care in an HMO. Jt Comm J Qual Improv 1996;22(10):673-82.

21. Rothberg MB, Wong JB. All dysuria is local. A cost-effectiveness model for designing site-specific management algorithms. J Gen Intern Med 2004; 19(5 Pt 1):433-43.

22. Kadiri S, Ajayi SO, Toki RA. Quinolones for shortterm treatment of uncomplicated urinary tract infection. East Afr Med J 1999;76(10):587-9.

23. Foxman B, Frerichs RR. Epidemiology of urinary tract infection: I. Diaphragm use and sexual intercourse. Am J Public Health 1985;75(11):1308-13.

24. Kugler JP, Yeash J, Rumbaugh PC. The impact of sociodemographic, health care system, and family function variables on prenatal care utilization in a military setting. J Fam Pract 1993;37(2):143-7.

25. Smoley BA, Smith NL, Runkle GP. Hypertension in a population of active duty service members. J Am Board Fam Med 2008;21(6):504-11.

26. Chang A. StatTools [computer software]. Available at: http://www.stattools.net/SSiz2props_Pgm.php. Accessed 10 July 2008.

27. Lenth RV (2006-9). Java applets for power and sample size [computer software]. Available at: http:// www.stat.uiowa.edu/ rlenth/Power. Accessed 10 July 2008. 


\section{Appendix}

\section{Table A1. Bacterial Resistance Rates}

\begin{tabular}{|c|c|c|c|c|}
\hline Organism* & Ampicillin & Nitrofurantoin & TMP/SMX & Ciprofloxacin \\
\hline \multicolumn{5}{|l|}{ Esherichia coli } \\
\hline$\% \mathrm{R}(\mathrm{n}=191)$ & 32 & 0.5 & 13 & 2 \\
\hline$\% \mathrm{IR}$ & 0.5 & 0.5 & 0 & 0.5 \\
\hline \multicolumn{5}{|l|}{ Klebsiella } \\
\hline$\% R(n=10)$ & 60 & 10 & 0 & 0 \\
\hline$\% \mathrm{IR}$ & 30 & 80 & 0 & 0 \\
\hline Staph sp. \%R (n = 9) & NT & 0 & 0 & 11 \\
\hline \multicolumn{5}{|l|}{ Proteus } \\
\hline$\% \mathrm{R}(\mathrm{n}=8)$ & 13 & 100 & 13 & 0 \\
\hline$\%$ IR & 0 & 0 & 0 & 13 \\
\hline Enterococcus \% R ( $\mathrm{n}=2)$ & 0 & 0 & 0 & 0 \\
\hline \multicolumn{5}{|l|}{ Citrobacter } \\
\hline$\% \mathrm{R}(\mathrm{n}=2)$ & 100 & 0 & 0 & 0 \\
\hline$\% \mathrm{IR}$ & 0 & 0 & 0 & 0 \\
\hline Enterobacter \% R ( $\mathrm{n}=1)$ & NT & IR & 0 & 0 \\
\hline Pseudomonas \% R ( $\mathrm{n}=1)$ & NT & NT & NT & 0 \\
\hline Salmonella \% R $(\mathrm{n}=1)$ & 0 & NT & 0 & 0 \\
\hline Strep $(\mathrm{n}=18)$ & NT & NT & NT & NT \\
\hline
\end{tabular}

${ }^{*}$ Escherichia coli was the most common pathogen, with 191 isolates (79\% of all isolates). Streptococcal (Strep) species were the second most common type of pathogen, with 18 isolates (7\%). There were 10 Klebsiella isolates (4\%), nine staphylococcal (Staph) species isolates (4\%), and eight Proteus isolates (3\%).

TMP-SMX, trimethoprim/sulfamethoxazole; NT, not tested; R, resistance; IR, intermediate resistance. 